\title{
Image Matching Algorithm Based on Contourlet Transform and VA Fast Search
}

\author{
Jie Fu, Pei Wang, Yan He \\ College of Mechanical and Electronic Engineering \\ Shanghai Normal University \\ Shanghai, China \\ shnu jiefu@hotmail.com
}

\author{
Xiao-qing $\mathrm{Xu}$ \\ School of Energy and Electrical \\ Hohai University \\ Nanjing, Jiangsu Province, China
}

\begin{abstract}
This article aim at the application of the high realtime required embedded terminal situation, an improved SIFT algorithm is proposed. After traditional SFIT algorithm get the stable points, global texture description was carried out on the key points of frequency domain by contourlet transform, select the top $1 \%$ of points, and then through a kind of nearest neighbor search algorithm based on Vector Angle (Vector Angle, VA) to match. This algorithm improves precision, speed and the accuracy of the embedded terminal.
\end{abstract}

Keywords- sift; contourlet; VA; Image matching

\section{INTRODUCTION}

In recent years, with 'The Internet of Things' concept put forward, such as image processing, computer vision, pattern recognition research has entered the stage of hot research direction again. Image matching technology is a focus in the field of its research content, the binocular vision, dynamic tracking, $3 \mathrm{~d}$ reconstruction has been widely used. Image matching technology is roughly divided into global and local match, global matching algorithm containing data information, high time complexity. It's hardly to achieve the goal of real-time, and difficult to achieve in the embedded terminal. However, local matching algorithm mainly extract interest point matching from left and right images, the result data greatly reduced, so the local matching algorithm in the embedded terminal developed rapidly. In the image matching based on local characteristics, the scale invariant feature transform(Scale Invariant Feature Transform, SIFT) ${ }^{[1]}$ algorithm is widely used. The key point detected from SIFT algorithm has good robustness, high positioning accuracy, repeatability etc. In the case of wide baseline, the rotation, scale, lighting and other geometrical invariability is well maintained, and high stability ${ }^{[2]}$. For real-time embedded terminal, SIFT algorithm has some shortcomings, such as the dimension of the feature descriptor is exorbitant, matching search strategy by exhaustive search will cause the time complexity of the algorithm is too high, affect the matching efficiency greatly. When there are multiple similar matching with the target area, SIFT feature vector is difficult to distinguish the performance, it can cause missing match.

Supported by the Program of Shanghai Normal University (DZLA7001-12-002006), Supported by Shanghai Normal University (SK20112)

\section{CONTOURLET - SifT FEATURES DESCRIPTION}

\section{A. Global information and Contourlet transform}

The features received from traditional SIFT, due to it ignored the relativity of position in the whole image, so that when there is a high degree of local information of similar characteristics, matching error probability is larger. So this paper will introduce a global information SIFT algorithm, supplement the SIFT features of traditional.

Contourlet transform[4] can effectively carries on the overall description of the texture for image's key areas in frequency domain. It is an efficient image representation method, and split the multi-scale analysis and direction analysis. It is a kind of transform with the multi-resolution, multi-scale, more directional and anisotropic properties, Basis function distribution on Multi-scale and Multidirection. A small amount of factor can effectively capture the image edge profile to approximate the original image and realizesparse expression of image signal. Contourlet transform is a kind of layer structure of filter bank made up by LP (Laplacian Pyramid, LP) and DFB (Directional Filter Bank, DFB). As shown in figure 1, different scales in LP is adopted in the first level of subband images, using DFB in grade 2 of high frequency subband images of each filter, achieve the purpose of to directional spectrum segmentation.

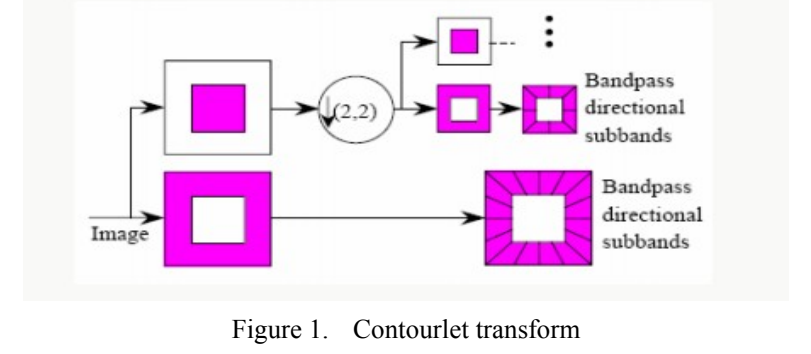

\section{B. Rotation invariant Contourlet character description}

A point $\mathrm{K}(x, y, \theta, \alpha)$ is detected from scale space, $(x, y)$ is the location of that point in the original image coordinates, $\theta$ is the direction of the point and $\sigma$ is scale parameters. According to the size of $\sigma$, right Angle coordinate system is established that is the Gaussian pyramid 
level corresponding to $\mathrm{k}$ as the center. Selecting neighborhood of $\mathrm{k}$ calculation in the neighborhood of each pixel gradient amplitude and gradient direction as follows:

$$
\begin{gathered}
m(x, y)=\left[(L(x+1, y)-L(x-1, y))^{2}+(L(x, y+1)-L(x, y-1))^{2}\right]^{1 / 2} \\
\theta(x, y)=\tan ^{-1}\{[L(x, y+1)-L(x, y-1)] /[L(x+1, y)-L(x-1, y)]\}
\end{gathered}
$$

Then the gradient direction histogram is generated, it counts the neighborhood pixels gradient direction. The direction of the gradient value range is $0^{\circ} \sim 360^{\circ}$, every $10^{\circ}$ a range, a total of 36 ranges. The main peak of gradient direction histogram corresponds to the main direction of the key points. The Gaussian image which corresponding to key point $k(x, y, \theta, \sigma)$, rotate to the reference direction according to the theta Angle, making the $\mathrm{k}$ with rotation invariance.

As shown in figure 2, after rotating image, we select the image area centered on $\mathrm{k}$, size is $2^{4} * 2^{4}, 2^{5} * 2^{5}, \ldots, 2^{t+3} * 2^{t+3}$, then execute contourlet character description respectively. We treat every square central area as a description the domain $\mathrm{P}_{\mathrm{i}}(\mathrm{i}=1,2, \ldots, \mathrm{t})$.

In this paper, we multistage decomposition of the test image as figure 3(a). We put the input signal that is decomposed into a low-frequency subband and 1 highfrequency subband by LP, high-frequency subband by DFB subband decomposition into a direction ( $\mathrm{j}$ for any positive integer, different resolution $\mathrm{j}$ may take different values). Contourlet decomposition produces a low frequency component and multiple high frequency subband per level, we then iterate process in the low frequency subband, the image can be realized in multi-resolution and multidirection decomposition (as shown in figure 3(b)). The Contourlet transform has good directivity and anisotropy.

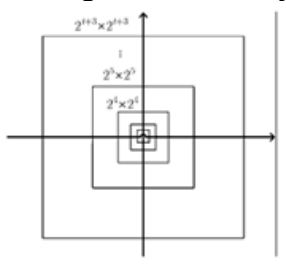

Figure 2. Choose Contourlet descriptor of building area
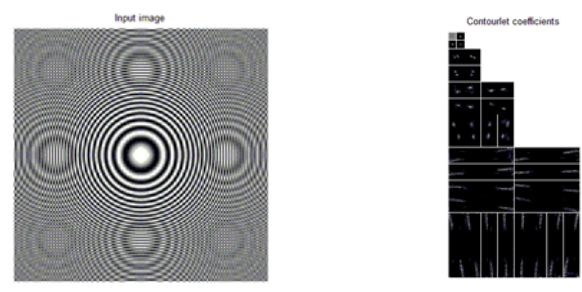

(a)the original image coefficient

Figure 3. The Contourlet transform of the image

Similar to figure 3, we carried out on the
$P_{i}(i=1,2, \cdots, t)$ contourlet transformation, We put the $P_{\mathrm{i}}$ decomposed into a series of direction, access to all levels of Contourlet direction subband coefficient of distribution, constructing the key $K$ feature vector. We calculate the direction of each subband decomposition coefficient of $\mu_{i}^{1}$ and standard $\delta_{i}^{1}$, which $\mathrm{M} * \mathrm{~N}$ is the size of the corresponding direction subband contourlet, L denotes the Lth subband direction as follows:

$$
\begin{aligned}
& \mu_{i}^{1}=\frac{1}{M^{*} N} \sum_{r=1}^{M} \sum_{c=1}^{N} \omega_{i}^{1}(r, c) \\
& \mu_{i}^{1}=\frac{1}{M^{*} N} \sum_{r=1}^{M} \sum_{c=1}^{N} \omega_{i}^{1}(r, c)
\end{aligned}
$$

Thus, we describing vector for the area $\mathrm{P}_{i}$ as follows:

$$
\text { Clet }_{i}=\left[\delta_{i}^{1}, \delta_{i}^{2}, \delta_{i}^{3}, \ldots, \delta_{i}^{m}\right]
$$

In which $\mathrm{m}$ denotes describe domain $\mathrm{P}_{i}$ through the contourlet transform, it was divided into $\mathrm{m}$ direction. The eigenvectors are normalized as follows:

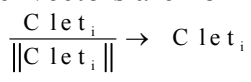

Says from the intuitive, I s smaller, the smaller the area, the more prominent the locality feature points, so the Clet $_{i}$ weighted, the weighted coefficient is as follows:

$$
\omega_{\mathrm{i}}=\exp \left[-\frac{(n-i)^{2}}{n}\right] /(2 \pi \varepsilon)
$$

The $\mathrm{n}$ is a positive integer that $2 \mathrm{n}$ is no greater than the image of arbitrary length, $\varepsilon$ is the selected constant. Therefore, the contourlet eigenvectors of the key point $\mathrm{k}$ can be expressed as follows:

$$
\text { Clet }=\left[\omega_{1}^{*} \operatorname{Clet}_{1}, \omega_{2} * \operatorname{Clet}_{2}, \omega_{3} * \operatorname{Clet}_{3}, \ldots, \omega_{t}^{*} \text { Clet }_{t}\right]
$$

\section{MATCHING ALGORITHM}

\section{A. VA rapid search strategy}

We assume that $\mathrm{d}$ dimensional space vector has two points, $\mathrm{P}_{1}=\left(\mathrm{x}_{1}, \mathrm{x}_{2}, \mathrm{x}_{3}, \ldots, \mathrm{x}_{\mathrm{d}}\right)$ and $\mathrm{P}_{2}=\left(\mathrm{y}_{1}, \mathrm{y}_{2}, \mathrm{y}_{3}, \ldots, \mathrm{y}_{\mathrm{d}}\right)$

Their corresponding vector as follows:

$$
\begin{aligned}
& V_{1}=\left[x_{1}, x_{2}, x_{3}, \ldots, x_{d}\right] \\
& V_{2}=\left[y_{1}, y_{2}, y_{3}, \ldots, y_{d}\right]
\end{aligned}
$$

The Euclidean distance between the point of $P_{1}$ and $P_{2}$ can be expressed as

$$
\mathrm{D}\left(\mathrm{P}_{1}, \mathrm{P}_{2}\right)=\sqrt{\left(x_{1}-y_{1}\right)^{2}+\left(x_{2}-y_{2}\right)^{2}+\cdots+\left(x_{d}-y_{d}\right)^{2}}
$$

The cosine theorem shows that vector and the Angle can be expressed as: 


$$
\begin{aligned}
& \left\langle\mathrm{V}_{\mathrm{P}} \mathrm{V}_{2}\right\rangle=\cos ^{-1} \frac{V_{1} \cdot V_{2}}{\left|V_{1}\right|\left|V_{2}\right|} \\
& =\cos ^{-1}\left(\frac{x_{1} y_{1}+x_{2} y_{2}+\cdots+x_{d} y_{d}}{\sqrt{x_{1}^{2}+x_{2}^{2}+\cdots+x_{d}^{2}} \sqrt{y_{1}^{2}+y_{2}^{2}+\cdots+y_{d}^{2}}}\right) \\
& \mathrm{D}\left(\mathrm{P}_{1} \mathrm{P}_{2}\right)=\sqrt{\left|\mathrm{V}_{1}\right|^{2}+\left|\mathrm{V}_{1}\right|^{2}-2 \mathrm{~V}_{1} \cdot \mathrm{V}_{2}}
\end{aligned}
$$

By contrast can be seen, when the length of the vector $V_{1}$ and $V_{2}$ is a certain, angle between them is smaller, the Euclidean distance $D\left(P_{1}, P_{2}\right)$ of points $P_{1}$ and $P_{2}$ which corresponding the vector $V_{1}$ and $V_{2}$ is smaller. Conversely, the Angle between the vector $V_{1}$ and $V_{2}$ is greater, the Euclidean distance $D\left(P_{1}, P_{2}\right)$ of points $P_{1}$ and $P_{2}$ is greater. When all the length of the vector are equal in the $F_{\mathrm{d}}$,

$$
\left\langle V_{\mathrm{i}}, V_{\mathrm{q}}\right\rangle=\cos ^{-1} \frac{V_{\mathrm{i}} \bullet V_{\mathrm{q}}}{\left|V_{\mathrm{i}}\right| V_{\mathrm{q}} \mid}
$$

Taking the minimum value of the vector must be the nearest neighbor of $K$. When the length of the vector is not equal in $F_{\mathrm{d}}$, above conclusion maybe not true. Taking the three dimensional Euclidean space for example which is shown in figures 4 (a), the Angle of vector $V_{2}$ and $V_{\mathrm{q}}$ is the minimum, but distance $d_{2}$ is more than the distance $d_{1}$ between vector $V_{1}$ and vector $V_{q}$. Therefore, the nearest neighbor is not $V_{2}$. In order to get real nearest neighbor closest to the $V_{q}$ as much as possible, we expand the search scope to all vectors in the collection. As shown in figure 4(b), if the search scope expanded to $\mathrm{K}$ as the axis, apex Angle of $2 \Delta \theta$ cone area, can get real nearest neighbor increasing $V_{1}$ with the increment of $\Delta \theta$, the probability of found nearest neighbor is also increased. Therefore, when $F_{d}$ is not equal to the length of the vector, we choose a suitable search scope $\Delta \theta$, the vector $V_{q}$ nearest neighbor still can be found.

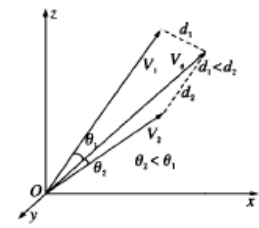

(a)The smallest included angle between $V_{2}$ and $V_{\mathrm{q}}$ not the nearest

$$
\text { neighbor of } V_{\mathrm{q}}
$$

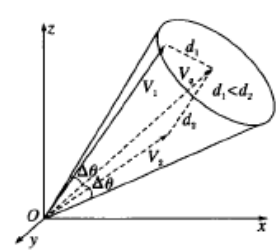

(b)The nearest neighbor $V_{1}$ successfully found by expanding search range

Figure 4. Schematic plot of nearest neighbor searching based on VA

\section{B. Recent search algorithm based on vector Angle}

Two vectors in a European space collection:

$\mathrm{F}_{\mathrm{d}}=\left\{\mathrm{V}_{\mathrm{i}} \mid \mathrm{V}_{\mathrm{i}} \in \mathrm{R}^{\mathrm{d}}, \mathrm{i}=1,2, \cdots, \mathrm{n}\right\}$

$\mathrm{F}_{\mathrm{d}}^{\prime}=\left\{\mathrm{V}_{\mathrm{q}} \mid \mathrm{V}_{\mathrm{q}} \in \mathrm{R}^{\mathrm{d}}, \mathrm{i}=1,2, \cdots, \mathrm{m}\right\}$

No need to search the nearest neighbor in the collection, the introduction of a random reference vector, and with the included Angle and deposited in the inventory in the array. So there is no need for calculation when searching and collection of all vector Angle, just calculate its Angle and the reference vector, and then search in the vector collection $F_{d}^{\prime \prime}=\left\{V_{i} \mid V_{i} \in F_{d}, V_{i}, V_{r} \in[\varphi+\Delta \theta, \varphi-\Delta \theta], i=1,2, \cdots, n\right\}$

Get real neighbors, as shown in figure 5, nearest neighbor falls in the three-dimensional space.

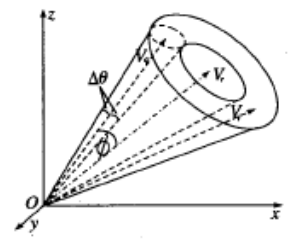

Figure 5. Schematic plot of nearest neighbor searching with VA after introducing a reference vector

\section{EXPERIMENT RESULTS}

This experiment is under the environment of MATLAB R2010b, we compared the description and matching effect between SIFT algorithm and Contourlet-SIFT algorithm; And we use the VA algorithm of this paper compared with feature points matching results of traditional SIFT.

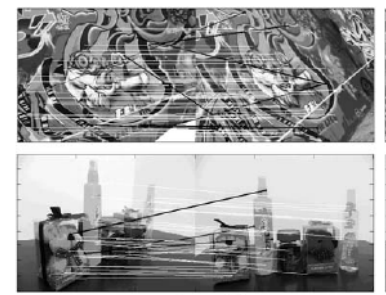

(a)SIFT

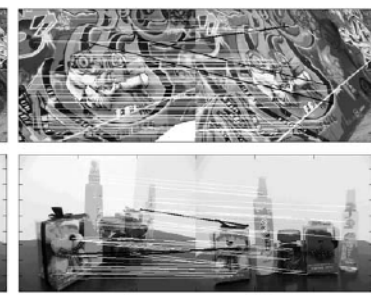

(b)My algorithm
Figure 6. the matching results under angle changes

Figure 6 shows the matching results under different 
Angle of view, when the threshold value of threshold consistent Contourlet-SIFT matching accuracy is not lower than SIFT, but the matching total increased. Thus it can be seen, in Contourlet texture filtering, not only on the similar area don't match, at the same time due to the frequency domain space Contourlet transform invariance has stable multiresolution and direction, through global texture feature compensation caused by perspective transformation of local information is missing, to match the rise in the Numbers.

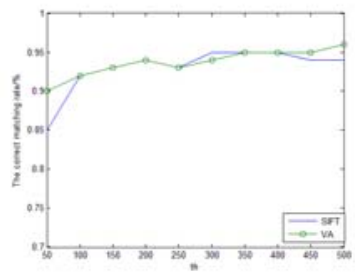

(a)Relationship between threshold threshold and correct matching ratio

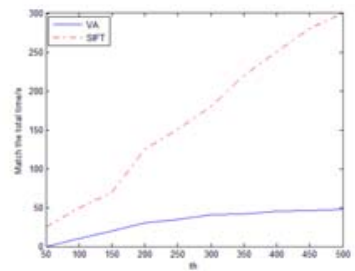

(b) Relationship between threshold threshold and total matching time

Figure 7. SIFT feature matching experimental results using VA and BBF algorithm

Figure 7(a) shows that using the VA and the BBF algorithm for correct matching rate of SIFT feature points matching with the same trend. Accuracy as the threshold increases when the threshold value is less than 200. When the threshold value more than 200 , the correct matching rate no longer increases, but tends to be stable. Can be seen from the figure, VA and correct matching rate of the $\mathrm{BBF}$ algorithm difference is very small, even more than the BBF algorithm is more stable. You can see by the (b), with the increasing of threshold value, VA and the BBF match the total time of the algorithm are the approximate linear growth trend, but the BBF algorithm to match the growth rate of total time about six times larger than the VA algorithm. From the above analysis shows that using VA algorithm to SIFT feature point matching can obtain satisfactory effect, and can significantly improve the matching speed.

\section{CONCLUSIONS}

In this paper, there is a algorithm based on contourlet transform and fast matching of VA search strategy, invariant to image scale airspace and frequency domain combining multiresolution representation, change the traditional feature vector representation method, finally the rapid search strategy based on vector angle matching theory has a lot to improve, and through the contrast test of different Angle analysis shows that the algorithm of this paper has greatly improved than before, and have a satisfactory result .

\section{ACKNOWLEDGMENT}

This work is Supported by the Program of Shanghai Normal University (DZLA-7001-12-002006), Supported by Shanghai Normal University (SK201127).

\section{REFERENCES}

[1] David G. Lowe. Distinctive Image Features from Scale-Invariant Key points $[\mathrm{J}]$. International Journal of Computer Vision. January 5,2004,60(2):91-110.

[2] Chen Shu rong, $\mathrm{Li}$ Bo. Contourlet-SIFT Feature Matching Algorithm[J]. Journal of Electronics \& Information Technology. May 2013,35(5):1215-1220.

[3] Ji Hua, Wu Yuan-hao, Sun Hong-hai, et al.. SIFT feature matching algorithm with global information[J]. Optics and Precision Engineering, 2009, 17(2): 439-444

[4] Do M N and Vetterli M. The contourlet transform: an efficient directional multiresolution image representation[J] IEEE Transactions on Image Processing, 2005, 14(12):2091-2106.

[5] LoweD G. Distinctive Image Features from Scale-Invariant Keypoints. International Journal of Computer Vision. 2004, 60(2) : 91110

[6] N Kingsbury. Complex Wavelets for Shift Invariant Analysis and Filtering of Signals[J]. Journal of Appl and Comput. Harmonic Analysis, 2001, 10 ( 3) : 2342253.

[7] A L Cunha, J Zhou, M N Do. The Non sub sampled Contourlet Trans2form: Theory, Design, and App lications[C]. IEEE Transactions on Image Processing, 2005.

[8] C. Hecker, B. Raabe, R.W. Enslow, J. DeWeese, J. Maynard, and K.van Prooijen, "Real-Time Motion Retargeting to Highly VariedUser-Created Morphologies," ACM Trans. Graphics, vol. 27, no. 3,2008.

[9] A. Buchanan and A.W. Fitzgibbon, "Interactive Feature TrackingUsing K-D Trees and Dynamic Programming," Proc. IEEE CSConf. Computer Vision and Pattern Recognition (CVPR), vol. 1,pp. 626-633, 2006.

[10] Scharstein, D., Szeliski, R.: A taxonomy and evaluation of dense twoframe stereocorrespondence algorithms. International Journal of Computer Vision 47 (2002)7-42 